\title{
On the Benefits of Argumentation-derived Evidence in Learning Policies
}

\author{
Chukwuemeka David Emele ${ }^{1}$, Timothy J. Norman ${ }^{1}$, \\ Frank Guerin ${ }^{1}$, and Simon Parsons ${ }^{2}$ \\ 1 University of Aberdeen, Aberdeen, AB24 3UE, UK \\ \{c.emele, t.j.norman, f.guerin\}@abdn.ac.uk \\ 2 Brooklyn College, City University of New York, 11210 NY, USA \\ parsons@sci.brooklyn. cuny.edu
}

\begin{abstract}
An important and non-trivial factor for effectively developing and resourcing plans in a collaborative context is an understanding of the policy and resource availability constraints under which others operate. We present an efficient approach for identifying, learning and modeling the policies of others during collaborative problem solving activities. The mechanisms presented in this paper will enable agents to build more effective argumentation strategies by keeping track of who might have, and be willing to provide the resources required for the enactment of a plan. We argue that agents can improve their argumentation strategies by building accurate models of others' policies regarding resource use, information provision, etc. In a set of experiments, we demonstrate the utility of this novel combination of techniques through empirical evaluation, in which we demonstrate that more accurate models of others' policies (or norms) can be developed more rapidly using various forms of evidence from argumentation-based dialogue.
\end{abstract}

\section{Introduction}

Distributed problem solving activities often require the formation of a team of collaborating agents. In such scenarios agents often operate under constraints placed on them by the organisations or interests that they represent. When these constraints are part of the standard operating procedures of the agents or the organisations in question, we refer to them as policies (also known as norms). Members of the team agree to collaborate and perform joint activities in a mutually acceptable fashion. Often, agents in the team represent different organisations, and so there are different organisational constraints imposed on them. Even within a single organisation, team members often represent suborganisations with different procedures and constraints. Examples of such constraints are those due to policies that guide the behaviour of representatives of organisations. Furthermore, team members may possess individual interests and goals that they seek to satisfy, which are not necessarily shared with other members of the team. These individual motivations largely determine the way in which members carry-out tasks assigned to them during joint activities. 
In this paper, we focus on policy and resource availability constraints, and define policy constraints as explicit prohibitions that members of the team are required to adhere to. Policy constraints may be team-wide or individual. We focus on individual policies. These policies are often private to that individual member or subset of the team, and are not necessarily shared with other members of the team. In order to develop effective plans, an understanding of the policy and resource availability constraints of other members in the team is beneficial. However, tracking and reasoning about such information is non-trivial.

Our conjecture is that machine learning techniques may be employed to aid decision making in this regard. Although this is not a new claim [7], it is novel to combine it with evidence derived from argumentation-based dialogue, which we call argumentation-derived evidence (ADE). We present a system where agents learn from dialogue by automatically extracting useful information (evidence) from the dialogue and using these to model the policies of others in order to adapt their behaviour in the future. We describe an experimental framework and present results of our evaluation in a resource provisioning scenario [5], which show empirically (1) that evidence derived from argumentation-based dialogue can indeed be effectively exploited to learn better (more complete and correct) models of the policy constraints that other agents operate within; and (2) that through the use of appropriate machine learning techniques more accurate and stable models of others' policies can be derived more rapidly than with simple memorisation of past experiences.

For example, consider the following snippet of dialogue that may occur between two agents $i$ and $j$ collaborating to hang a picture [11].

\begin{tabular}{|l||l|}
\hline Example 1: & Example 2: \\
\hline$i$ : Can I have a screw-driver? & $i$ : Can I have a screw-driver? \\
$j:$ What do you want to use it for? & $j:$ What do you want to use it for? \\
$i:$ To hang a picture. & $i:$ To hang a picture. \\
$j:$ No. & $j:$ I can provide you with a hammer instead. \\
& $i$ I accept a hammer. \\
\hline
\end{tabular}

Following from the interaction in example 1, there is very little that we can learn from the encounter. It is unclear why agent $j$ said no to agent $i$ 's request. It could be that there exists some policy $\mathrm{X}$ that forbids agent $j$ from providing the screw-driver to agent $i$ or it could be that the screw-driver is not available at the moment. On the other hand, suppose we have an argumentation framework that allows agents to suggest alternatives as in example 2 or ask for and receive explanations as in examples 3 and 4 , then agent $i$ can gather more evidence regarding the provision of the resources involved.

Considering examples 3 and 4 , it is worth noting that without the additional evidence, obtained by the information-seeking dialogue, the two cases are indistinguishable. This means that the agent will effectively be guessing which class these cases fall into. The additional evidence allows the agent to learn the right 


\begin{tabular}{|l||l|}
\hline Example 3: & Example 4: \\
\hline$i:$ Can I have a screw-driver? & $i$ : Can I have a screw-driver? \\
$j:$ What do you want to use it for? & $j:$ What do you want to use it for? \\
$i$ : To hang a picture. & $i$ : To hang a picture. \\
$j:$ No. & $j:$ No. \\
$i:$ Why? & $i:$ Why? \\
$j:$ I'm not permitted to release the screw-driver. & $j:$ Screw-driver is not available. \\
\hline
\end{tabular}

classification for each of the cases. It should be noted here that although in example 3, we now have a statement that the resource is not to be provided for policy reasons, the question remains: what are the important characteristics of the prevailing circumstances that characterise this policy?

In a domain where there are underlying constraints that could yield similar results, standard machine learning techniques will have limited efficacy. Using argumentation to gather additional evidence could improve the accuracy of the information learned about the policies of others. We claim that significant improvements can be achieved because argumentation can help clarify reasons behind decisions made by the provider.

In the research presented in this paper, we intend to validate the following hypotheses: (1) Allowing agents to exchange arguments during practical dialogue (like negotiation) will mean that the proportion of correct policies learned during interaction will increase faster than when there is no exchange of arguments.

(2) Through the use of appropriate machine learning techniques more accurate and stable models of others' policies can be derived more rapidly than with simple memorisation of past experiences.

The remainder of this paper is organised as follows: In section 2 we briefly describe argumentation-based dialogue and introduce the negotiation protocol employed. Learning policies is discussed in section 3 and section 4 describes our simulation environment. Experimental results are reported in section 5 and section 6 discusses related work and future direction. The paper is concluded in section 7 .

\section{Argumentation-based Dialogue}

In this section we present the argumentation-based negotiation protocol which will be used in guiding the negotiation process, and for obtaining additional evidence from the interaction. This protocol uses information-seeking dialogue [17] to probe for additional evidence.

\subsection{The Negotiation Protocol}

The negotiation for resources takes place in a turn-taking fashion, where the seeker agent sends a request for resource to a provider agent. Figure 1 captures the negotiation protocol in a AUML-like interaction diagram (www.fipa.org). If the provider agent has the requested resource in its resource pool and it is in a 
usable state then it checks whether there is any policy constraint that forbids it from providing the resource to the seeker or not. Peradventure, the provider agent needs more information from the seeker in order to make a decision, the provider agent would ask for more information to be provided. This is the information gathering stage. The information gathering cycle will continue until the provider has acquired enough information (necessary to make the decision), or the seeker refuses to provide more information and the negotiation ends.

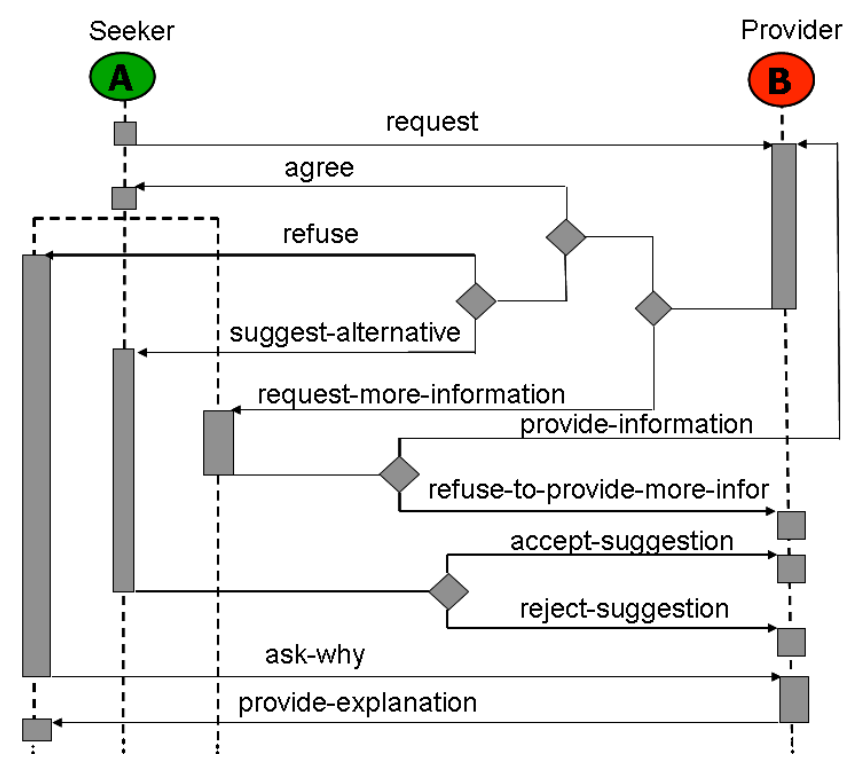

Fig. 1. The negotiation protocol.

The provider agent releases the resource to the seeker agent if there is no policy that prohibits the provider agent from doing so. Otherwise, the provider agent offers an alternative resource (if there are no policies that forbid that line of action and the alternative resource is available). When an alternative resource is suggested by the provider agent, the seeker agent evaluates it. If it is acceptable, the seeker agent accepts it and the negotiation ends. Otherwise, the seeker agent refuses the alternative (in principle, this cycle may be repeated until an alternative is accepted or the negotiation ends). However, for simplicity and brevity, only one suggest-refuse cycle is permitted per request.

From a learning point of view, the suggestion of alternative resources is a positive evidence that the provider agent does not have any policy that forbids the provision of the alternative resource to the seeker. In addition, it provides an evidence that the alternative resource is also available. This extra evidence, we anticipate, may help to improve the performance of the learner in predicting the policy constraints of the provider agents in future encounters. 
If there is a policy constraint that forbids the provision of the resource, or the resource is not available then the provider agent will refuse to provide the resource to the seeker agent. From the seeker's perspective, the refusal could be as a result of policy constraint or because the resource is not available. In order to disambiguate which of these constraints are responsible for the refusal, the seeker agent switches to argumentation based dialogue. The seeker agent asks for explanations for the refusal so as to gather further evidence and thereby identify the underlying constraints. The provider agent, therefore, responds with some explanations and the negotiation ends. Three categories of explanations are possible in this framework: (1) Policy constraints (2) Resource not available (3) Won't tell you. These evidence will be explored in the following section.

\subsection{Argumentation-derived Evidence}

Following the argumentation-based negotiation protocol described earlier, the agents could ask for more information (with respect to a request or the response to a request), which indicates what constraints others may be operating within. For instance, let us assume that a provider agent has a policy that forbids it from providing a screw-driver to any seeker agent that intends to use it for hanging a picture. Then, whenever a screw-driver is requested the provider agent will probe for more information to ascertain that the purpose the seeker intends to use the screw-driver for is not hanging a picture. This extra evidence could be useful. Similarly, whenever a seeker agent's request is refused then the seeker agent will ask for explanations/justifications for the refusal. These additional evidence are beneficial, and we expect them to improve the quality of the models of other agents that can be inferred in future encounters.

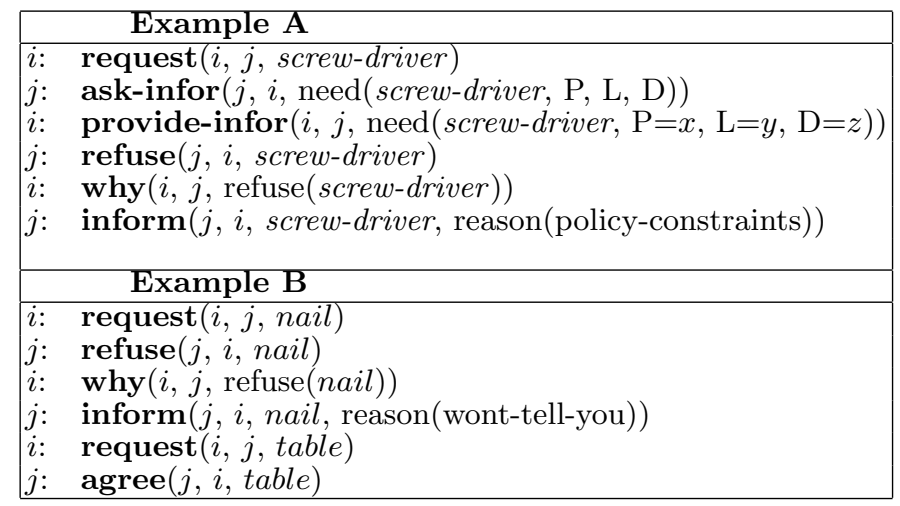

Fig. 2. Dialogue snippets between agents $i$ and $j$

Figure 2 shows two simple examples of the kind of dialogue that may occur between two agents, $i$ and $j$. For the purpose of the example, we use need $(R, \mathbf{P}$, $\mathbf{L}, \mathbf{D})$ to denote that the seeker agent intends to use the resource $R$ for purpose 
$\mathrm{P}$ at location $\mathrm{L}$ on day $\mathrm{D}$. Note that although this is presented as a dialogue between two agents, in reality the initiator (agent $i$, the agent that wishes to resource its plan) may engage in multiple instances of this dialogue with other agents.

\section{Learning Policies}

In this section we discuss the machine learning techniques that we have explored for learning policies through argumentation-derived evidence. These techniques include decision tree learning (C4.5), instance-based learning ( $k$-Nearest Neighbours, abbreviated as $k$-NN) and rule-based learning (Sequential Covering, abbreviated as SC).

Our technique does not attempt to replace machine learning nor compete with existing techniques. Rather, we seek ways to combine argumentation analysis with already existing machine learning techniques with a view to improving the performance of agents at predicting the policy constraints of others. We anticipate that this could enable them to build more effective argumentation strategies. In other words, we argue that evidence derived from argumentationbased dialogue can indeed be effectively exploited to learn better (more complete and correct) models of the policy constraints that other agents operate within. Also, we claim that through the use of appropriate machine learning techniques more accurate and stable models of others' policies can be derived more rapidly than with simple memorisation of past experiences. In future encounters, the seeker agent attempts to predict the policies of the provider agent based on the model it has built.

\subsection{Decision Tree Learning (C4.5)}

C4.5 [13] builds decision trees from a set of training data, using the concept of information entropy [8] (beyond the scope of this paper). Generally, the training data is a set $S=s_{1}, s_{2}, \ldots, s_{n}$ of already classified samples. Each sample $s_{i}=$ $x_{1}, x_{2}, \ldots, x_{m}$ is a vector where $x_{1}, x_{2}, \ldots, x_{m}$ represent attributes of the sample. The training data is augmented with a vector $C=c_{1}, c_{2}, \ldots, c_{n}$ where $c_{1}, c_{2}, \ldots, c_{n}$ represent the class to which each sample belongs.

Integrating this algorithm into our system with the intention of learning policies is appropriate since the algorithm supports concept learning and policies can be conceived as concepts/features of an agent. Agent policies are represented as a vector of attributes (e.g. resource, purpose, location, etc.) and these attributes are communicated back and forth during negotiation. The $\mathrm{C} 4.5$ algorithm is then used to classify each set of attributes (policy instance) into a class. There are two classes: grant and deny. Grant means that the provider agent will possibly provide the resource that is requested while deny implies that the provider agent will potentially refuse. The leaf nodes of a decision tree hold the class labels of the instances while the non-leaf nodes hold the test attributes. In order to classify a test instance, the $\mathrm{C} 4.5$ algorithm searches from the root node by 
examining the value of test attributes until a leaf node is reached and the label of that node becomes the class of the test instance.

The problem with this algorithm is that it is not incremental, which means all the training examples should exist before learning. To overcome this problem, the system keeps track of the provider agent's responses (both positive and negative instances). After a number of interactions, the decision tree is rebuilt. Without doubt, there is a computational drawback involved in periodically reconstructing the decision tree. However, in practice, we have evaluated C4.5 to be fast and the reconstruction cost to be small.

The $\mathrm{C} 4.5$ algorithm has three base cases.

- All the samples in the list belong to the same class. When this happens, it simply creates a leaf node for the decision tree saying to choose that class.

- None of the features provide any information gain. In this case, C4.5 creates a decision node higher up the tree using the expected value of the class.

- Instance of previously-unseen class encountered. Again, C4.5 creates a decision node higher up the tree using the expected value.

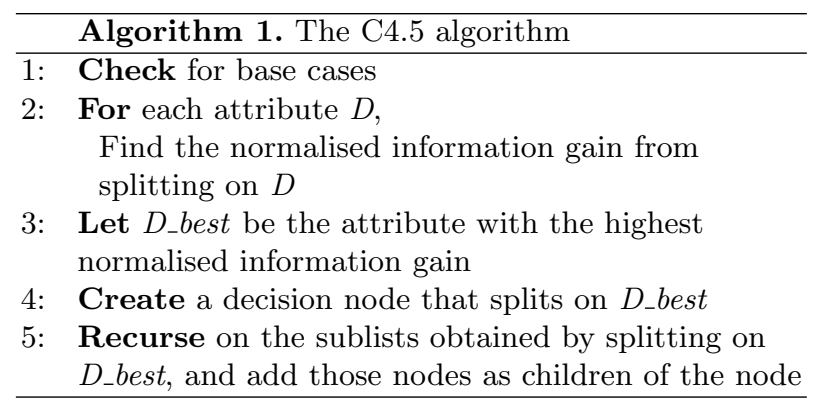

Fig. 3. The C4.5 algorithm.

\subsection{Instance-based Learning ( $k$-NN)}

The $k$-nearest neighbours algorithm ( $k$-NN) [3] is a type of instance-based learning, or lazy learning, where the function is only approximated locally and all computation is deferred until classification. The universal set of all the policies an agent may be operating within could be conceived as a feature space (or a grid) and the various policy instances represent points on the grid. Using $k$-NN, a policy instance is classified by a majority vote of its neighbours, with the policy instance being assigned to the class most common amongst its $k$ nearest neighbours, where $k$ is a positive integer, typically small. The $k$-NN algorithm is incremental, which means all the training examples need not exist at the beginning of the learning process. This is a good feature because the policy model could be updated as new knowledge is learned. 
The $k$-nearest neighbour algorithm is sensitive to the local structure of the data and this, interestingly, makes $k$-NN a good candidate for learning policies because slight changes in the variables/attributes of a policy could trigger different action. For example:

Policy1: You are permitted to release resource $R$ to team member $X$ if his affiliation is $O$ and $R$ is to be deployed at location $L$ for purpose $P$ on day 1 .

Policy2: You are prohibited from releasing resource $R$ to team member $X$ if his affiliation is $O$ and $R$ is to be deployed at location $L$ for purpose $P$ on day 2.

In order to identify neighbours, the policy instances are represented by position vectors in a multidimensional feature space. In this approach, new policy instances are classified based on the closest training examples in the feature space. A policy instance is assigned to the class $c$ if it is the most frequent class label among the $k$ nearest training samples. It is usual to use the Euclidean distance, though other distance measures, such as the Manhattan distance, Hamming distance could in principle be used instead. The training phase of the algorithm consists only of storing the feature vectors and class labels of the training samples. In the actual classification phase, the test sample (whose class is not known) is represented as a vector in the feature space. Distances from the new vector to all stored vectors are computed and $k$ closest samples are selected.

A major drawback to using this technique to classify a new vector to a class is that the classes with the more frequent examples tend to dominate the prediction of the new vector, as they tend to come up in the $k$ nearest neighbours when the neighbours are computed due to their large number. The distance-weighted $k$-NN algorithm, which weights the contribution of each of the $k$ neighbours according to their distance to the new vector, uses distance weights to minimise the bias caused by the imbalance in the training examples by giving greater weight to closer neighbours. In our work, the weight of a neighbour is computed as the inverse of its distance from the new vector.

\subsection{Rule-based Learning (Sequential Covering)}

Since policies guide the way entities within a community (or domain) act by providing rules for their behaviour it makes sense to learn policies as rules. Sequential covering algorithm $[8,2]$ is a rule-based learning technique, which constructs rules by sequentially covering the examples. The sequential covering algorithm, $\mathrm{SC}$ for short, is a method that induces one rule at a time (by selecting attributevalue pairs that satisfy the rule), removes the data covered by the rule and then iterates the process. SC generates rules for each class by looking at the training data and adding rules that completely describe all tuples in that class. For each class value, rule antecedents are initially empty sets, augmented gradually for covering as many examples as possible. Figure 4 outlines the sequential covering algorithm in pseudo-code.

In this study we used three different machine learning mechanisms: Decision tree learning, Instance-based learning and Rule-based learning. These three 


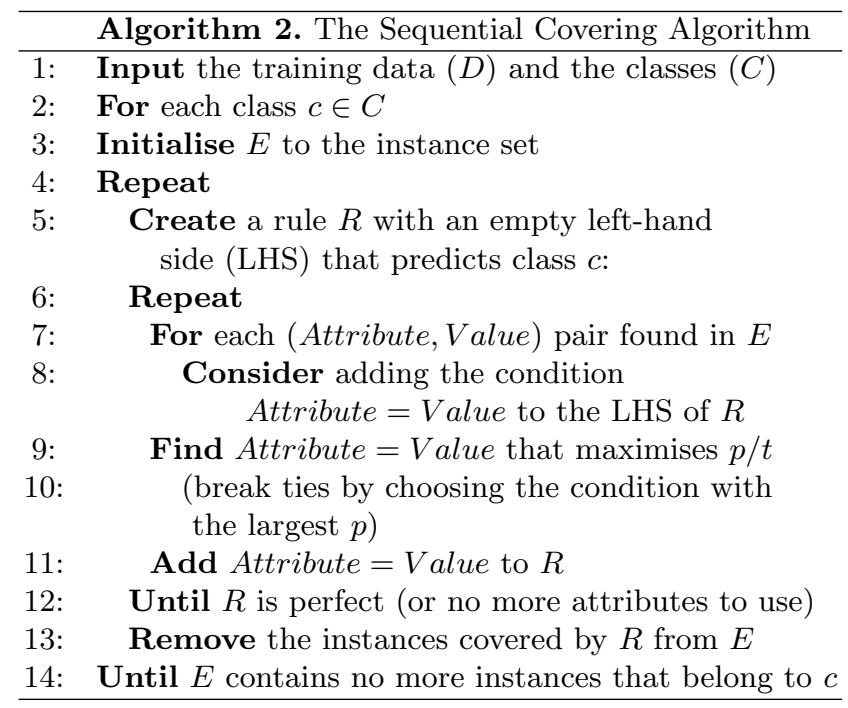

Fig. 4. The Sequential Covering Algorithm.

mechanisms represent very different classes of machine learning algorithms. The rationale for exploring a range of learning techniques is to demonstrate the utility of argumentation-derived evidence regardless of the machine learning technique employed. Thus, we hypothesize that the use of evidence acquired through argumentation significantly improves the performance of machine learning in the development and refinement of models of other agents. Also, we claim that through the use of appropriate machine learning techniques more accurate and stable models of others' policies can be derived more rapidly than with simple memorisation of past experiences.

\section{Simulation Environment}

To test our hypotheses, we developed a simulation environment that combines mechanisms for agents to engage in argumentative dialogue and to learn from dialogical encounters with other agents. For the purpose of resourcing plans, agents may act as resource seekers, which collaborate and communicate with potential providers to perform joint actions. The enactment of both seeker and provider roles are governed by individual policies that regulate their actions. A seeker agent requires resources in order to carry out some assigned tasks. The seeker agent generates requests in accordance with its policies and negotiates with the provider agents based on these constraints. On the other hand, provider agents have access to certain resources and may have policies that govern the provision of such resources to other members of the team.

Although agents may have prior assumptions about the policies that constrain the activities of others, these models are often incomplete and may be 
inaccurate. Provider agents do not have an unlimited pool of resources and so some resources may be temporarily unavailable. By a resource being available we mean that it is not committed to another task (or agent) at the time requested and the resource is in a usable state. Both seeker and provider agents have access to the team-wide policies but not the individual policies of others. Agents in this domain play the role of a seeker or a provider in different interactions.

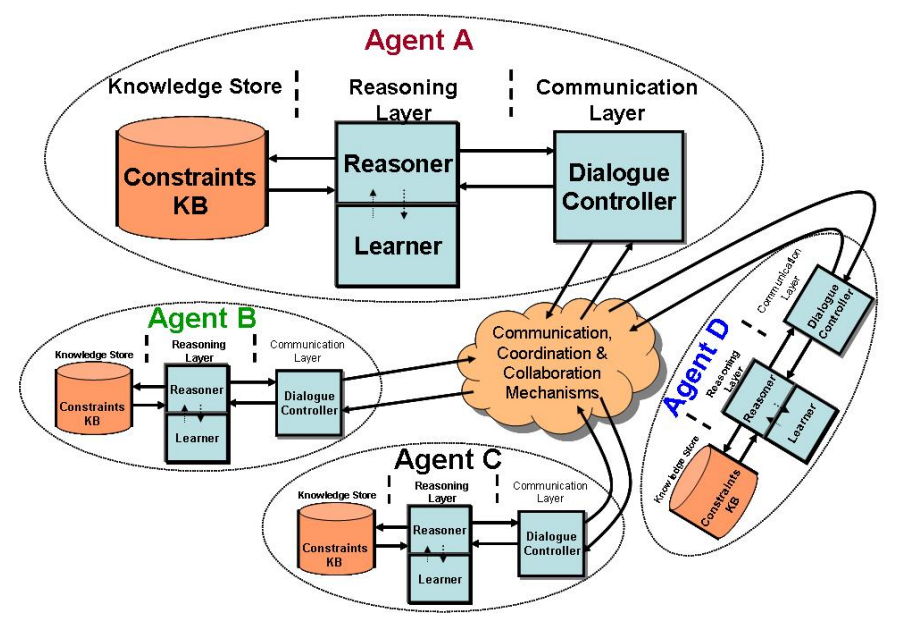

Fig. 5. Architecture of the framework for learning policies in team-based activities using dialogue.

\subsection{Architecture}

Figure 5 depicts our architecture. Each agent has two main layers, the communication layer and the reasoning layer. The communication layer embodies the dialogue controller, which handles all communication with other agents in the domain. The dialogue controller sends/receives messages to/from other agents, and the reasoning layer reasons over the dialogue. If an agent is playing the role of a seeker agent then the dialogue controller sends out the request for resources. On the other hand, if the agent is a provider agent then the dialogue controller receives a request and passes it on to the reasoning layer.

The reasoning layer consists of two modules: the reasoner and the learner. Upon receiving a message (e.g. a request), the reasoner evaluates the message and determines the response of the agent. In most cases, the reasoner looks up policy constraints from the knowledge-base and generates the appropriate response for the agent. Policy and non-policy constraints are stored in the constraints knowledge-base. Whenever the agent observes a new pattern of behaviour the agent uses this experience as evidence for learning, and updates the model of the other agent accordingly. The learner uses standard machine learning techniques 
to learn policies based on the perceived actions of other agents. The learning techniques are discussed in Section 3.

The knowledge store in Figure 5 acts as a repository where an agent stores the constraints it has learned by interacting with other agents in the domain. The information includes the features that an agent requires in order to make a decision about providing a resource or not. For example, following from [11], a provider agent $B$ may need to know what the purpose for requesting a screwdriver is before deciding whether to release the screw-driver or not. The seeker agent stores such information about agent $B$ in the knowledge store. Also, the decision of $B$ after the purpose has been revealed will also be learned for future interactions.

To achieve this, we have developed a simple dialogue game ${ }^{3}$ involving seeker agents and provider agents operating under different constraints. The players take turns and the game starts with an agent, $i$, sending a request to another agent, $j$, for the use of some resources needed to fulfill a plan. The other agent $(j)$ responds with an agree or refuse based on the prevailing context, e.g. policy constraints. The requesting agent could ask for explanations and reasons for an action, and so on until the game ends.

\subsection{Implementation}

We implemented a simulation environment for agent support in team-based problem solving and integrated our learning and argumentation mechanisms into the framework. The policies are encoded as rules in a rule engine [6]. The application programming interface in Weka [18] was used to integrate standard machine learning algorithms into the framework. We note that, although these three learning algorithms were used, the framework is configured such that other machine learning algorithms can be plugged in. As discussed in the previous section, we evaluated the performance of a decision tree learner (C4.5), an Instance based learner ( $k$-Nearest Neighbour algorithm) and a rule based learner (Sequential Covering) in learning policies through argumentation-derived evidence.

The simulation environment allows us to generate multiple providers with randomised policies, seeker agents with randomised initial models of the policies of providers in the simulation and randomised problems for the seeker to solve (that is, random resource requirements). The seeker predicts (based on the model of the provider) whether the provider has a policy that forbids/permits the provision of such resource in that context. The seeker requests the required resource from the provider agent and the provider uses a simple decision function (See Figure 6) to decide whether to grant or deny the request.

If the decision of the provider agent deviates from the predictions of the seeker agent then the seeker agent seeks additional evidence (through dialogue) to disambiguate whether the deviation was as a result of policy or resource availability constraints. The dialogue follows the protocol specified in Figure 1,

\footnotetext{
${ }^{3}$ Dialogue games have proven extremely useful for modeling various forms of reasoning in many domains [1].
} 
and at the end of the interaction the outcome is learned by the seeker and the model of the provider is updated accordingly. This adaptive learning process serves to improve the quality of the models of the other agents that can be inferred from their observable actions in future interactions.

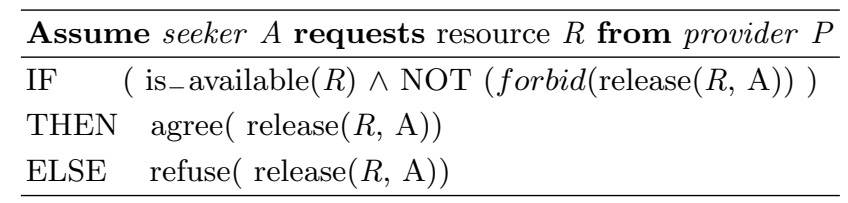

Fig. 6. Provider agents' pseudo decision function

\section{$5 \quad$ Experiments and Results}

In a series of experiments, we show how learning techniques and argumentation can support agents engaging in collaborative activities, increase their predictive accuracy, avoid unnecessary policy conflicts, hence improve their performance. The experiments show that agents can effectively and rapidly increase their predictive accuracy of the learned model through the use of dialogue.

The scenario adopted in this research involves a team of five software agents (one seeker and four provider agents) collaborating to complete a joint activity in a region over a period of three days. The region is divided into five locations. There are five resource types, and five purposes that a resource could be used to fulfill. A task involves the seeker agent identifying resource needs for a plan and collaborating with the provider agents to see how that plan can be resourced.

Argumentation-derived evidence (ADE) was incorporated into the learning process of the three machine learning techniques $(\mathrm{C} 4.5, k-\mathrm{NN}$, and $\mathrm{SC})$ described earlier, and their performances in learning the policy constraints of others were evaluated. A simple lookup table (hereafter called, LT) was used as a control condition and it serves as a structure for simple memorisation of outcomes from past encounters.

\subsection{Results}

This section presents the results of the experiments carried out to validate this work. Experiments were conducted with seeker agents initialised with random models of the policies of provider agents. 100 runs were conducted for each case, and tasks were randomly created during each run from 375 possible configurations.

Table 1 illustrates the effectiveness of identifying and learning policies through argumentation-derived evidence using the three machine learning techniques described earlier, and the control condition (lookup table). It shows the average 
Table 1. Average percentage of policies classified correctly and standard deviation

\begin{tabular}{|c|c|c|c|c|c|c|}
\hline Approach $\quad$ Tasks & 1000 & 2000 & 3000 & 4000 & 5000 & 6000 \\
\hline LT-ADE & $65.1 \pm 6.5$ & $70.3 \pm 10.3$ & $75.6 \pm 6.7$ & $78.1 \pm 10.2$ & $79.3 \pm 8.3$ & $81.3 \pm 10.1$ \\
\hline LT+ADE & $66.3 \pm 6.0$ & $79.3 \pm 9.3$ & $83.6 \pm 8.2$ & $81.7 \pm 11.2$ & $81.4 \pm 7.8$ & $84.7 \pm 9.1$ \\
\hline C4.5-ADE & $58.3 \pm 15.1$ & $69.2 \pm 16.6$ & $75.1 \pm 12.0$ & $82.1 \pm 12.3$ & $85.3 \pm 8.9$ & $88.2 \pm 8.2$ \\
\hline $\mathrm{C} 4.5+\mathrm{ADE}$ & $60.3 \pm 14.4$ & $75.0 \pm 12.6$ & $83.6 \pm 6.5$ & $89.9 \pm 5.2$ & $93.0 \pm 3.4$ & $95.6 \pm 5.1$ \\
\hline$k$-NN-ADE & $65.2 \pm 9.8$ & $71.0 \pm 7.8$ & $75.3 \pm 5.3$ & $80.7 \pm 3.8$ & $81.0 \pm 4.1$ & $82.0 \pm 3.8$ \\
\hline$k-\mathrm{NN}+\mathrm{ADE}$ & $71.1 \pm 9.0$ & $85.9 \pm 7.3$ & $92.0 \pm 4.6$ & $96.8 \pm 3.1$ & $97.3 \pm 3.6$ & $98.4 \pm 1.7$ \\
\hline SC-ADE & $66.7 \pm 8.2$ & $71.7 \pm 6.0$ & $78.7 \pm 8.4$ & $84.3 \pm 6.5$ & $87.4 \pm 6.0$ & $90.6 \pm 5.3$ \\
\hline $\mathrm{SC}+\mathrm{ADE}$ & $67.7 \pm 7.7$ & $87.1 \pm 6.4$ & $94.1 \pm 4.2$ & $96.6 \pm 4.1$ & $97.5 \pm 2.6$ & $99.2 \pm 1.0$ \\
\hline
\end{tabular}

percentage of policies classified correctly and the standard deviations for each of the approaches, namely: Lookup Table without the aid of argumentation-derived evidence (LT-ADE), Lookup Table enhanced with argumentation-derived evidence (LT+ADE), C4.5-ADE, C4.5+ADE, $k$-NN-ADE, $k$-NN+ADE, SC-ADE, and SC+ADE. In each case, the model of others' policies is recomputed after each set of 1000 tasks. For all three machine learning techniques considered, the percentage of policies predicted correctly as a result of exploiting evidence derived from argumentation was consistently and significantly higher than those predicted without such evidence. Figure 7 gives a graphical illustration of the effectiveness of learning policies with the aid of argumentation-derived evidence using rule-based learning technique, for instance. After 3000 tasks, the accuracy of the approach with additional evidence had risen above $94 \%$ while the configuration without additional evidence was approaching $79 \%$. It is easy to see that the experiments where additional evidence was combined with machine learning significantly and consistently outperformed those without additional evidence. These results show that the exchange of arguments during practical dialogue enabled agents to learn and build more accurate models of others' policies much faster than scenarios where there was no exchange of arguments.

Figure 8 captures the effectiveness of the three machine learning techniques described earlier, and a simple memorisation technique (a lookup table) in learning policies. The result shows that both instance-based learning $(k-\mathrm{NN}+\mathrm{ADE})$ and rule-based learning (SC+ADE) constantly and consistently outperform the control condition (LT+ADE) throughout the experiment. It is interesting to see that, with relatively small training set, the control condition performed better than the decision tree learner (C4.5+ADE). This is, we believe, because the model built by the decision tree learner overfit the data. The tree was pruned after each set of 1000 tasks and after 3000 tasks the accuracy of the C4.5+ADE model rose to about $83 \%$ to tie with the control condition and from then the decision tree learner performed better than the control condition. The performance of the control condition dropped to about $81 \%$ after 4000 tasks. After 6000 tasks the accuracy of the decision tree learner had risen above $95 \%$ while that of the control condition was just over $84 \%$. 


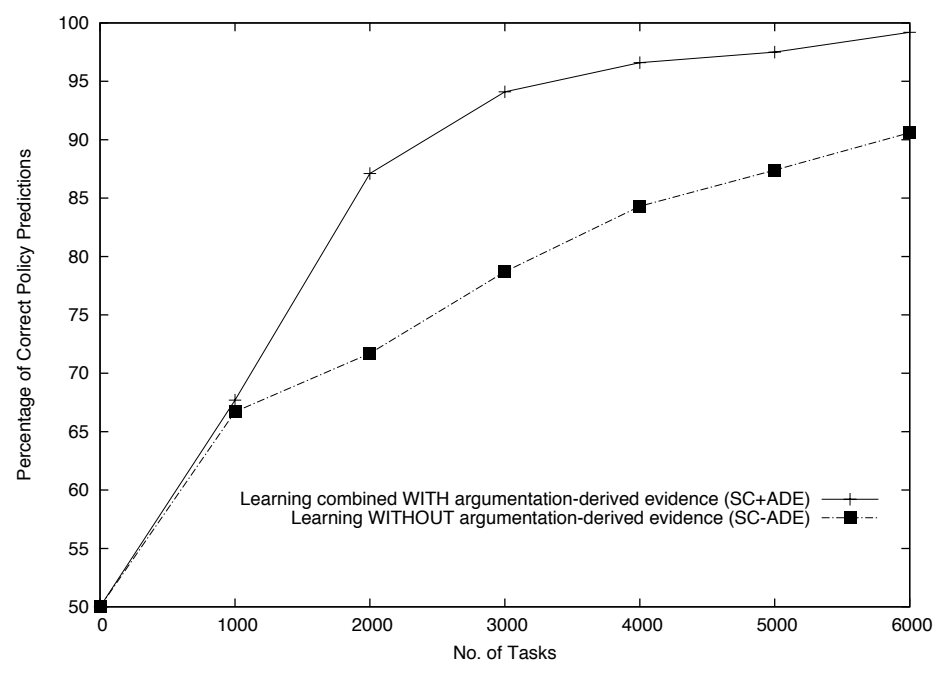

Fig. 7. Graph showing the effectiveness of allowing the exchange of arguments in learning policies.

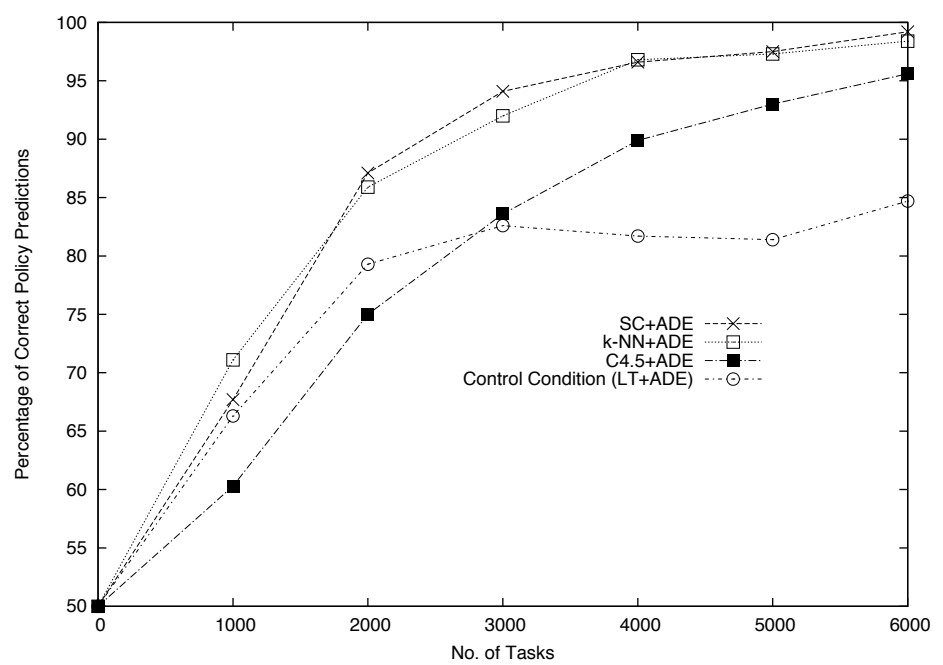

Fig. 8. Graph showing the effectiveness of learning policies with the aid of argumentation-derived evidence using various techniques (LT+ADE, C4.5+ADE, $k$ $\mathrm{NN}+\mathrm{ADE} \& \mathrm{SC}+\mathrm{ADE})$.

Tests of statistical significance were applied to the results. The standard deviations of the results were analysed and the trend line plotted. (See Figure 9). 
Using linear regression, the analysis of variance (ANOVA) shows that as the number of tasks increases, each of the three machine learning techniques (with or without argumentation-derived evidence) consistently converges with a $95 \%$ confidence interval. Furthermore, for all the pairwise comparisons, the scenarios where argumentation-derived evidence was combined with machine learning techniques consistently yielded higher rates of convergence $(p<0.02)$ than those without additional evidence. Specifically, the decision tree learner enhanced with argumentation-derived evidence (C4.5+ADE) converges $(y=15.3944-0.0022 x)$ with a $F$ value of 15.66 and significance $p=0.0167$. The $k$-NN+ADE converges $(y=9.7983-0.0014 x)$ with a $F$ value of 38.58 and significance $p=0.0034$, and the $\operatorname{SC}+\operatorname{ADE}(y=8.819-0.0013 x)$ converges with a $F$ value of 136.45 and significance $p=0.0003$. On the other hand, with a significance $p=0.3957$, there is no statistical significance as to whether LT+ADE converges or not. These results confirm our hypotheses.

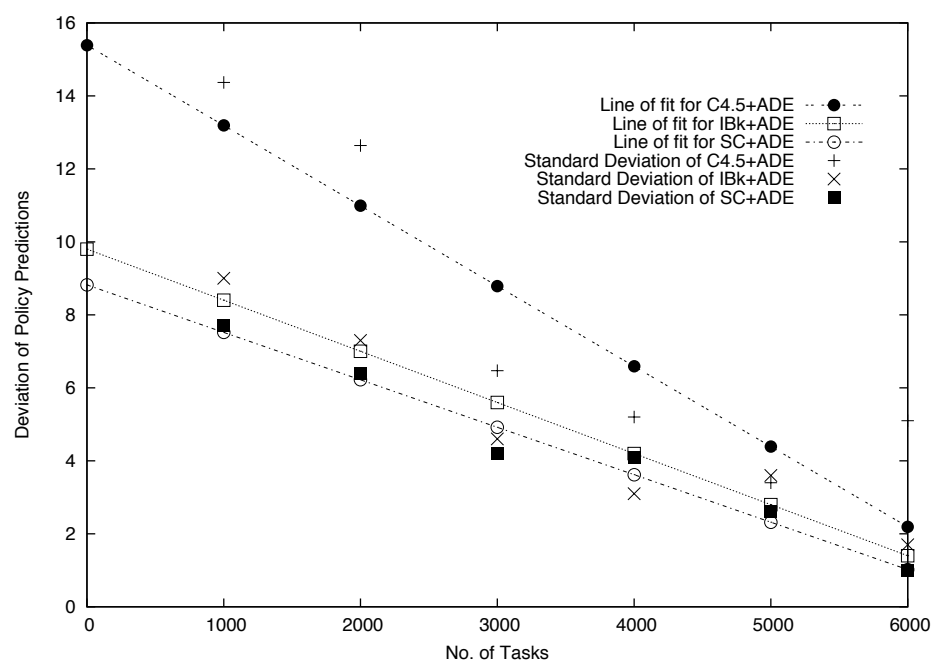

Fig. 9. Graph showing the rate of convergence of the three techniques enhanced with $\mathrm{ADE}$ in learning policies (C4.5+ADE, $k$-NN+ADE, \& $\mathrm{SC}+\mathrm{ADE})$.

\section{Discussion and Related Work}

The research presented in this paper represents the first model for using evidence derived from argumentation to learn underlying social characteristics (e.g. policies/norms) of others. There is, however, some prior research in combining machine learning and argumentation, and in using argument structures for 
machine learning. In that research, Možina et al. [9] propose a novel inductionbased machine learning mechanism using argumentation. The work implemented an argument-based extension of $\mathrm{CN} 2$ rule learning (ABCN2) and showed that ABCN2 out-performed CN2 in most tasks. However, the framework developed in that research will struggle to disambiguate between constraints that may produce similar outcome/effect, which is the main issue we are addressing in our work. Also, the authors assume that the agent knows and has access to the arguments required to improve the prediction accuracy, but we argue that it is not always the case. As a result, we employ information-seeking dialogue to tease out evidence that could be used to improve performance.

In related research, Rovatsos et al. [15] use hierarchical reinforcement learning in modifying symbolic constructs (interaction frames) that regulate agent conversation patterns, and argue that their approach could improve an agent's conversation strategy. In our work, we used information-seeking dialogue to obtain evidence from the interaction and learned the entire sequence as against a segment (frame) of the interaction [15]. We have demonstrated the effectiveness of using argumentation-derived evidence to learn underlying social characteristics (e.g. policies) without assuming that those underlying features are public knowledge.

In recent research, Sycara et al. [16] investigate agent support for human teams in which software agents aid the decision making of team members during collaborative planning. One area of support that was identified as important in this context is guidance in making policy-compliant decisions. This prior research focuses on giving guidance to humans regarding their own policies. An important and open question, however, is how can agents support human decision makers in developing models of others' policies and using these in guiding the decision maker? Our work is aimed at bridging this gap (a preliminary version was presented in [4]). We employ a novel combination of techniques in identifying, learning and building accurate models of others' policies, with a view to exploiting these in supporting human decision making.

In other research, Rahwan et al. [14] present a formal framework for analysing the outcomes of interest-based negotiation (IBN) dialogues and established that providing further information (especially about underlying interests) improves the likelihood and quality of an outcome. Policy constraints can be captured as underlying goals that agents are hoping to achieve (by adhering to them) and so argumentation can be used to tease out information regarding those constraints. In circumstances where knowledge is incomplete or imperfect, argumentation has proven to be effective in reaching some goals that would have otherwise been unreachable. It is worth noting that our work differs from Rahwan et al. [14] in that while the authors are interested in gathering meta-information and using it to support interest-based negotiation, we are interested in learning the policies that other agents are operating within and using this knowledge to guide how a plan is resourced. Also, the authors evaluated the work via examples rather than rigorous experimental investigation. Our framework neatly combines machine 
learning and argumentation in predicting what others' policies are. Furthermore, our work is aimed at supporting human decision making in team-based activities.

In our future work, we plan to develop strategies for advising human decision makers on how a plan may be resourced and who to talk to on the basis of policy and resource availability constraints learned [10]. Parsons et al. [12] investigated the properties of argumentation-based dialogues and examined how different classes of protocols can have different outcomes. Furthermore, we plan to explore ideas from this work to see which class of protocol will yield the "best" result in this kind of task. We are hoping that some of these ideas will drive the work on developing strategies for choosing who to talk to.

\section{Conclusions}

In this paper, we have presented a technique that combines machine learning and argumentation for learning policies in a team of collaborating agents engaging in joint activities. We believe, to the best of our knowledge, that this is the first study into learning models of other agents using argumentation-derived evidence. The results of our empirical investigations show that evidence derived from argumentation can have a statistically significant positive impact on identifying, learning and modeling others' policies during collaborative activities. The results also demonstrate that through the use of appropriate machine learning techniques more accurate and stable models of others' policies can be derived more rapidly than with simple memorisation of past experiences. Accurate policy models can inform strategies for advising human decision makers on how a plan may be resourced and who to talk to [16], and may aid in the development of more effective strategies for agents [10]. Our results demonstrate that significant improvements can be achieved by combining machine learning techniques with argumentation-derived evidence. Having shown that accurate models of others' policies could be learned through argumentation-derived evidence, we conjecture that one could, in principle, learn accurate models of other agents' properties (e.g. priorities, preferences, and so on).

\section{Acknowledgements}

This research was sponsored by the U.S. Army Research Laboratory and the U.K. Ministry of Defence and was accomplished under Agreement Number W911NF06-3-0001. The views and conclusions contained in this document are those of the author(s) and should not be interpreted as representing the official policies, either expressed or implied, of the U.S. Army Research Laboratory, the U.S. Government, the U.K. Ministry of Defence or the U.K. Government. The U.S. and U.K. Governments are authorized to reproduce and distribute reprints for Government purposes notwithstanding any copyright notation hereon. 


\section{References}

1. T. J. M. Bench-Capon, J. B. Freeman, H. Hohmann, and H. Prakken. Computational models, argumentation theories and legal practice. In C. Reed and T. J. Norman, editors, Argumentation Machines. New Frontiers in Argument and Computation, pages 85-120, Dordrecht, The Netherlands, 2003. Kluwer Academic Publishers.

2. J. Cendrowska. Prism: An algorithm for inducing modular rules. International Journal of Man-Machine Studies, 27(4):349-370, 1987.

3. T. Cover and P. Hart. Nearest neighbor pattern classification. IEEE Transaction on Information Theory, 13(1):21-27, 1967.

4. C. D. Emele, T. J. Norman, F. Guerin, and S. Parsons. Learning policies through argumentation-derived evidence (extended abstract). In van der Hoek, Lesprance, Kaminka, Luck, and Sen, editors, Proc. of 9th Int. Conf. on Autonomous Agents and Multiagent Systems (AAMAS 2010), Toronto, Canada, 2009. To appear.

5. C. D. Emele, T. J. Norman, F. Guerin, and S. Parsons. Learning policy constraints through dialogue. In Proc. of the AAAI Fall Symposium on The Uses of Computational Argumentation, pages 20-26, Virginia, USA, 2009.

6. E. Friedman-Hill. Jess in Action. Manning, 2003.

7. A. Kelemen, Y. Liang, and S. Franklin. A comparative study of different machine learning approaches for decision making. In N. E. Mastorakis, editor, Recent Advances in Simulation, Computational Methods and Soft Computing, pages 181-186, Piraeus, Greece, 2002. WSEAS Press.

8. T. M. Mitchell. Machine Learning. McGraw Hill, 1997.

9. M. Možina, J. Žabkar, and I. Bratko. Argument based machine learning. Artificial Intelligence, 171(10-15):922-937, 2007.

10. N. Oren, T. J. Norman, and A. Preece. Loose lips sink ships: A heuristic for argumentation. In Proc. of the 3rd Int'l Workshop on Argumentation in MultiAgent Systems (ArgMAS 2006), pages 121-134, 2006.

11. S. Parsons and N. R. Jennings. Negotiation through argumentation-A preliminary report. In Proc. of the 2nd Int'l Conference Multi-Agent Systems (ICMAS'96), pages 267-274, Kyoto, Japan, 1996.

12. S. Parsons, M. Wooldridge, and L. Amgoud. Properties and complexities of some formal inter-agent dialogues. Journal of Logic and Computation, 13(3):347-376, 2003.

13. J. R. Quinlan. C4.5: programs for machine learning. Morgan Kaufmann Publishers Inc., San Francisco, CA, USA, 1993.

14. I. Rahwan, P. Pasquier, L. Sonenberg, and F. Dignum. On the benefits of exploiting underlying goals in argument-based negotiation. In Proc. of the 22nd International Conference on Artificial Intelligence (AAAI), California, USA, 2007. AAAI Press.

15. M. Rovatsos, I. Rahwan, F. Fischer, and G. Weiss. Practical strategic reasoning and adaptation in rational argument-based negotiation. In Argumentation in MultiAgent Systems, volume 4049 of LNCS, pages 122-137. Springer-Berlin, 2005.

16. K. Sycara, T. J. Norman, J. A. Giampapa, M. J. Kollingbaum, C. Burnett, D. Masato, M. McCallum, and M. H. Strub. Agent support for policy-driven collaborative mission planning. The Computer Journal, page bxp061, 2009.

17. D. N. Walton and E. C. W. Krabbe. Commitment in Dialogue: Basic Concepts of Interpersonal Reasoning. SUNY Press, Albany, NY, USA, 1995.

18. I. H. Witten and E. Frank. Data Mining: Practical machine learning tools and techniques. Morgan Kaufmann, San Francisco, 2nd edition, 2005. 